\title{
Validation of the English Language Pain Sensitivity Questionnaire
}

\author{
Alethia Baldwin Sellers, MD, * Ruth Ruscheweyh, MD, $\dagger$ Bernard Joseph Kelley, MD, $\neq$ \\ Timothy J. Ness, MD, PhD, * and Thomas R. Vetter, MD, MPH*
}

\begin{abstract}
Background and Objectives: The Pain Sensitivity Questionnaire (PSQ) is predictive of pain-related responses to experimental stimuli in German-speaking individuals. Here, we explored the validation of the English translation of the PSQ (PSQ-E).

Methods: One hundred thirty-six patients scheduled to undergo a low back interventional procedure completed the PSQ-E and other questionnaires including the Brief Pain Inventory. Pain ratings on a visual analog scale (VAS) were obtained following 2 standardized injections of subcutaneous lidocaine (VAS 1, infiltration in hand; VAS 2, infiltration of procedural site). The VAS measures were compared with the PSQ-E data and other inventories using linear regression analysis with stepwise selection of variables.

Results: The PSQ-E properties were in all respects similar to those of the original German PSQ. VAS 1 magnitude was predicted by PSQE-minor $(r=0.26, P<0.01)$. VAS 2 magnitude was predicted by PSQ-E-minor $(r=0.34, P<0.001)$, and the prediction was significantly enhanced by further inclusion of the Brief Pain Inventory interference score (total $r=0.40, P<0.001$ ).

Conclusions: The study demonstrated that a significant correlation exists between the PSQ-E and clinically relevant pain ratings. This study validates the PSQ-E both in terms of measuring pain sensitivity and as possible means of recognizing patients with high pain sensitivity. Defining this subset of patients may have clinical utility in the future.
\end{abstract}

(Reg Anesth Pain Med 2013;38: 508-514)

C hronic pain patients often undergo interventional procedures as a part of their treatment. Many biopsychosocial factors contribute to the pain experienced by a patient during and after such an interventional procedure. ${ }^{1-3}$ Patients' innate pain sensitivity is one factor likely to influence their perception of procedure-related pain. ${ }^{4,5}$ Presently, there are a limited number of validated tools to assess this factor before the performance of a procedure.

Experimental determination of innate pain sensitivity using quantitative sensory testing techniques is of interest because of clinical applications in predicting procedural pain and treatment response. ${ }^{4,5}$ Preoperative experimental pain sensitivity is a predictor of acute postoperative pain, ${ }^{6}$ and heightened

From the *Department of Anesthesiology, University of Alabama School of Medicine, Birmingham, AL; †Department of Neurology, University of Munich, Munich, Germany; and $\ddagger$ Department of Anesthesiology, Charles George VA Medical Center, Asheville, NC.

Accepted for publication July 24, 2013.

Address correspondence to: Alethia Baldwin Sellers, MD, Department of Anesthesiology, University of Alabama School of Medicine,

JT862, 619 19th Street South, Birmingham, AL 35249-6810 (e-mail: abaldwin@uab.edu).

The authors declare no conflict of interest

Supplemental digital content is available for this article. Direct URL citations appear in the printed text and are provided in the HTML and PDF versions of this article on the journal's Web site (www.rapm.org).

Copyright (C) 2013 by American Society of Regional Anesthesia and Pain Medicine

ISSN: 1098-7339

DOI: 10.1097/AAP.0000000000000007 experimental pain sensitivity has been observed in numerous chronic pain disorders including low back pain. ${ }^{7}$ Stimuli of different types (thermal, mechanical, ischemic, or electrical) have been applied in the experimental assessment of pain sensitivity. ${ }^{8} \mathrm{Un}$ fortunately, the utility of such methodologies is limited by a number of factors, including the need for specialized equipment; increased staffing time and costs; and the aversive experience for the tested participants. ${ }^{9}$ The Pain Sensitivity Questionnaire (PSQ) was developed as a clinically useful, valid self-rating measure of pain sensitivity without these limitations. The PSQ has been demonstrated to provide information similar to experimentally derived pain sensitivity assessments. ${ }^{9}$ Specifically, the PSQ scores have been shown in a German cohort to correlate significantly with a variety of experimentally produced pain intensity ratings, both in healthy participants and chronic pain patients. ${ }^{9,10}$ The PSQ has yet to be studied in a native English-speaking population or applied in a clinical pain management setting.

Innate pain sensitivity may affect the need for intravenous (IV) sedatives and analgesics during an interventional pain procedure and the response to the pain treatment intervention. There is also an experimental finding that chronic pain patients who exhibit high pain sensitivity respond less well to treatment than those with lower pain sensitivity. ${ }^{1,12}$ The greater procedural pain perceived by some patients may contribute to overall treatment failure by reducing their willingness to participate in additional interventions. Thus, if patients have a high level of innate pain sensitivity, successful interventional pain treatment may require procedural sedation and analgesia. The PSQ is a lowrespondent-burden instrument that may help identify patients who are highly sensitive.

Because the English version of the PSQ (PSQ-E) (Supplemental Digital Content 1, http://links.lww.com/AAP/A94) may have clinical utility in the future, this study was undertaken in a clinical pain management setting to validate the questionnaire in an adult chronic pain population.

\section{METHODS}

\section{Setting and Participants}

This study was conducted in an outpatient chronic pain medicine clinic between May 2010 and July 2011. This study was approved by the institutional review board of the University of Alabama at Birmingham and abided by the Ethical Principles for Medical Research Involving Human Subjects outlined in the Declaration of Helsinki. Written patient consent and authorization of use of protected health information were obtained before study enrollment. Study participation was offered to adult chronic low back pain patients (having pain for greater than 3 months) who were scheduled to receive a lumbar epidural steroid injection (LESI), lumbar facet joint injection (LFJI), or sacroiliac joint injection (SIJI). Exclusion criteria included failed back surgery syndrome (postlaminectomy syndrome, lumbar region, International Classification of Diseases, Ninth 
Revision: 722.83), morbid obesity (body mass index, $>40$ ), being a non-English speaker, allergy to an amide local anesthetic, and history of a seizure disorder, diabetic neuropathy, substance abuse disorder, or major psychiatric disorder (eg, severe depression, bipolar disorder, Axis II personality disorder, and schizophrenia). In addition, only white subjects were included in the present study for improved comparability with existing data collected with the German version of the PSQ. All subjects reported to be English speakers born in the United States. A parallel study examined the effects of ethnic/cultural differences on these measures.

\section{Study Design}

This observational study was prospective and single arm in design. On the day on which participants were to undergo their interventional pain procedure, each completed the Brief Pain Inventory-Short Form (BPI-SF), Roland-Morris Back Pain Questionnaire (RMQ), Hospital Anxiety and Depression Scale (HADS), Pain Catastrophizing Scale (PCS), and PSQ. Patient age, sex, race, primary chronic pain-related diagnosis, duration of chronic pain symptoms, and chronic opioid and nonopioid medication use were extracted from the study participant's medical record.

\section{Patient Measurement Instruments}

\section{Visual Analog Scale}

The visual analog scale (VAS) for pain intensity has been widely applied in pain management and clinical research ${ }^{13,14}$ The VAS used was a horizontal $100-\mathrm{mm}$ line, anchored by verbal descriptors "no pain" (pain score of 0) and "worst pain" (pain score of 100), along which patients were asked to record their pain intensity.

\section{Brief Pain Inventory-Short Form}

The BPI-SF is a self-administered questionnaire that assesses the severity of pain and the impact of pain on daily function (ie, functional disability), ${ }^{15}$ using an 11-point numerical rating scale with anchors of "no pain" and "pain as bad as you can imagine." The BPI-SF also measures how much pain has interfered with 7 daily activities in the last 24 hours, specifically, general activity, walking, work, mood, enjoyment of life, relations with others, and sleep. ${ }^{15}$ Respondents rate each activity on an 11-point scale (from 0, "does not interfere" to 10 , "completely interferes"). The BPI-SF pain interference value was scored here as typically the mean of the 7 interference items, with a scoring range of 0 to 10 .

\section{Roland-Morris Back Pain Questionnaire}

The RMQ was created by selecting 24 pain and function items from the Sickness Impact Profile considered to be relevant to chronic low back pain. ${ }^{16-18}$

\section{Hospital Anxiety and Depression Scale}

The HADS has been widely applied in inpatient and outpatient health care settings to detect symptoms of anxiety and depression in nonpsychiatric patients. ${ }^{19}$

\section{Pain Catastrophizing Scale}

The PCS validly and reliably assesses the construct of pain catastrophizing. For research analysis purposes, a study participant's PCS score can be used as a confounding covariate and/or a primary or secondary outcome measure. ${ }^{20}$

\section{Pain Sensitivity Questionnaire}

The PSQ is a low-respondent burden, patient self-completed, pencil-and-paper instrument for the assessment of pain sensitivity and is intended to provide information similar to experimentally derived pain sensitivity assessment. ${ }^{9}$ Comprising 17 items (rated from 0 , not at all painful to 10 , most severe pain imaginable) and requiring 5 to 10 minutes to complete, the PSQ is based on pain-intensity ratings of daily life situations. Three items describe situations that are normally not rated as painful by healthy individuals (eg, taking a warm shower) and do not form part of the final score. In addition to a PSQ total score, a PSQ minor score and a PSQ moderate score are generated. ${ }^{9}$ These 2 subscores were identified in the previous study ${ }^{9}$ by factor analysis, each including 7 items that on average were rated as moderately painful (mean rating of 4-6 on the 11point-scale, PSQ-moderate) or as causing minor pain (mean rating of $<4$, PSQ-minor). The PSQ-total score was calculated as the average rating of items $1,2,3,4,6,7,8,10,11,12,14,15$, 16 , and 17 (all but the 3 nonpainful items), the PSQ-minor score was calculated as the average rating of items $3,6,7,10,11,12$, and 14 and the PSQ-moderate score was calculated as the average rating of items $1,2,3,8,15,16$, and $17 .{ }^{9}$ The original German version of the PSQ has been shown in a German cohort to be significantly correlated to a variety of experimentally produced pain intensity ratings, both in healthy controls and in chronic pain patients. ${ }^{9,10}$ The previously published English translation of the PSQ (PSQ-E) was used in the present study. ${ }^{9}$ This translation was performed according to the guidelines for cross-cultural adaptation of self-report measures. ${ }^{21}$ Two bilingual, native English speakers independently translated the original German version of PSQ. The 2 translated versions and the original version were compared and discussed by the translators until consensus was reached. Then, back translation was performed by 2 independent bilingual, native German speakers, both professional translators who were unaware of the purpose of translation. The back translation was compared with the original German version and discussed by an expert committee comprised of all 4 translators, the developer, a psychologist with experience in questionnaire design and cross-cultural adaptation, and several health care professionals until reaching consensus.

\section{Lidocaine as Experimental Pain Stimulus}

The present study used the injection of subcutaneous lidocaine as a controllable, clinically relevant noxious stimulus in a fashion similar to previous clinical studies. ${ }^{22-26}$ The sensations evoked by a subcutaneous lidocaine stimulus have sufficient reproducibility to allow for statistical comparison between alternate treatment groups and to demonstrate differences with relatively small numbers of subjects. ${ }^{27-29}$ Most pertinent to our study, the subcutaneous infiltration of lidocaine has been used as a standardized experimental pain stimulus to assess hyperalgesia ${ }^{30}$ and verbal and behavioral pain responses ${ }^{31}$ in patients undergoing interventional pain treatment procedures for chronic low back pain. A recent report suggests this may be useful even as a predictor of outcomes. ${ }^{32}$

\section{Study Protocol}

After informed consent and completion of questionnaires, patients had an IV catheter placed in the dorsum of their hand. Before IV placement, patients received $1 \mathrm{~mL}$ of nonbuffered, plain $1 \%$ lidocaine as a subcutaneous local anesthetic using a 25-gauge hypodermic needle. Each participant then immediately rated the pain associated with this injection (VAS 1) before 
the insertion of the IV catheter. Patients were then placed in the prone position on a fluoroscopic procedure table. Fluoroscopic guidance was used to identify the site of the injection. A skin wheal was created there with another $1 \mathrm{~mL}$ of nonbuffered, plain $1 \%$ lidocaine delivered via a 25 -gauge hypodermic needle, and participants were asked immediately to rate the pain associated with the second injection (VAS 2). Patients then received $2 \mathrm{mg}$ of IV midazolam, $8 \mathrm{~mL}$ of buffered, plain $1 \%$ lidocaine, via a 22-gauge hypodermic needle, into the deeper soft and ligamentous tissues at the site of their LESI, LFJI, or SIJI, and the procedure was performed. During the LESI, an 18-gauge 3.5-in Touhy needle was advanced under anteroposterior (AP) and lateral fluoroscopy using the loss of resistance technique. One milliliter of contrast was injected in the lateral and AP position to confirm epidural contrast spread and lessen the likelihood of vascular uptake. A mixture of a local anesthetic, lidocaine, and steroid, triamcinolone, or depomedrol, was injected without difficulty. During the LFJIs and SIJIs, a 22-gauge 3.5-in spinal needle was advanced under AP, lateral, and oblique fluoroscopy. When the needle tip was visualized in the joint space, $0.5 \mathrm{~mL}$ of contrast was injected to confirm intra-articular and periarticular contrast spread and lessen the likelihood of vascular uptake. A mixture of local anesthetic, bupivacaine, and steroid, triamcinolone, or depo-medrol, was injected without difficulty. All patients were then transferred to the postprocedure care area and subsequently given standard clinical care.

\section{Statistical Analyses}

A power analysis was conducted using GPower software (version 3.1.2; Kiel, Germany) to determine the number of participants included in the study. We assumed that the correlations between PSQ scores and procedural pain ratings would be smaller than those found with experimental pain testing $(0.5-0.7)^{9,10}$ because of greater interindividual variability, due to larger group inhomogeneity, different procedures used, and different physicians carrying out these procedures. We therefore powered the study to detect a correlation of Pearson $r$ greater than or equal to 0.25 at $P<0.05$ and with a power of 0.8 , which implicates that a sample size of 120 participants or more is needed. In the present study, we recruited 136 participants.

Continuous variables were summarized using descriptive statistics, giving mean (SD). Results of the psychological questionnaires and VAS ratings were treated as continuous variables for the purpose of the present paper. Categorical variables (sex, type of intervention, medication use) were reported as frequency counts and percentages. Normality was tested using the Kolmogorov-Smirnov test. Several parameters (PSQ minor scores, RMQ scores, clinical pain duration, VAS 1 and VAS 2 scores) were not normally distributed because of significant skewness in their distribution. Although the magnitude of parametric coefficients (eg, Pearson $r$ ) is robust against deviations from normality, significance levels and confidence intervals may be invalid for nonnormal distributions. On the other hand, nonparametric tests give valid significance levels in the absence of normality but the magnitude of coefficients (eg, Spearman $\rho$ ) is difficult to interpret (eg, in terms of shared variance) ${ }^{33}$ We therefore report parametric coefficients for correlations with these parameters but verified significance levels by calculating the corresponding nonparametric $P$ value in every case. Pearson $r$ was used to test for correlations in all other cases. Bonferroni adjustment was used to correct for multiple testing. Analysis of variance was used to test for the influence of the type of interventional pain procedure on pain ratings. Pearson $r$, linear regression analysis, and analysis of variance were used to identify demographic and clinical predictors of procedural pain. For all analyses, a $P$ value of less than 0.05 was considered significant. All statistical analyses were performed using SPSS Statistics, version 18.0 (IBM; Somers, New York).

\section{RESULTS}

A total of 136 patients were enrolled in this study. This included a convenience sample of patients with low back pain scheduled for a low back interventional procedure and patients who passed the exclusion criteria. A flow diagram is presented (Supplemental Digital Content 2, http://links.lww.com/AAP/A95). The demographics and clinical characteristics of this sequential convenience sample are presented in Table 1.

\section{Properties of the PSQ-E}

Scores of the English PSQ (PSQ-E) are reported in Table 2. There were no significant sex differences in PSQ-E scores. There was a significant correlation between age and PSQ-Etotal $(0.19, P<0.05)$ and between age and PSQ-E-minor ( $r=0.20, P<0.05$; also significant using Spearman $\rho ; P<0.05$ ) but not between age and PSQ-E-moderate. However, these correlations did not survive Bonferroni correction.

Among the psychological cofactors, PSQ-E scores were significantly correlated with pain catastrophizing (PCS scores) but not with anxiety or depression (HADS scores, Table 3).

Among the clinical pain characteristics, PSQ-E scores were significantly correlated with BPI pain scores, but correlations with BPI interference scores, Roland-Morris scores or clinical pain duration did not reach significance (Table 3 ).

TABLE 1. Characteristics of the Study Population $(n=136)$

\begin{tabular}{lc}
\hline Age, $\mathrm{y}$ & $54(14)$ \\
Sex & \\
$\quad$ Female & $61.0 \%(\mathrm{n}=83)$ \\
$\quad$ Male & $39.0 \%(\mathrm{n}=53)$ \\
Medication & \\
$\quad$ Opioids [oral morphine & $55.0 \%(\mathrm{n}=75)[26.8(61.3) \mathrm{mg}]$ \\
$\quad$ equivalents/d] & $35.3 \%(\mathrm{n}=48)$ \\
Psychotropic drugs* & $41.5 \%(\mathrm{n}=56)$ \\
Antiepileptic drugs & \\
$\quad$ for pain therapy) & $70(78)$ \\
Duration of pain, mo & $6.0(1.7)$ \\
BPI pain score [0-10] & $6.3(2.3)$ \\
BPI interference score [0-10] & $15.3(5.2)$ \\
Roland-Morris score [0-24] & $7.4(4.0)$ \\
HADS-D [0-21] & $8.7(4.3)$ \\
HADS-A [0-21] & $23.1(13.1)$ \\
PCS [0-52] & \\
Procedure & $60.7 \%(\mathrm{n}=82)$ \\
LESI & $8.1 \%(\mathrm{n}=11)$ \\
LFJI & $31.1 \%(\mathrm{n}=42)$ \\
SIJI &
\end{tabular}

Age, pain duration, morphine equivalents, and questionnaire scores are given as mean (SD). For categorical variables, percentages and absolute values are given.

*Antidepressants, neuroleptics, and benzodiazepines.

HADS-A, anxiety subscore; HADS-D, depression subscore. 
TABLE 2. PSQ-E Scores

\begin{tabular}{lccc}
\hline & & \multicolumn{2}{c}{ Sex } \\
\cline { 3 - 4 } & Total & Female & Male \\
\hline $\mathrm{n}$ & 136 & 83 & 53 \\
PSQ-E-total & $4.2(2.0)$ & $4.3(2.0)$ & $4.0(2.0)$ \\
PSQ-E-minor & $3.2(2.0)$ & $3.3(2.0)$ & $2.9(2.0)$ \\
PSQ-E-moderate & $5.2(2.1)$ & $5.3(2.2)$ & $5.0(2.0)$ \\
\hline
\end{tabular}

Values are mean (SD).

Scores were not significantly different in males and females.

\section{Prediction of Pain With Lidocaine Infiltration}

Lidocaine infiltration pain ratings on the VAS (0-100) were 23 (22) at VAS 1 (infiltration of lidocaine in dorsum of hand for IV placement) and 35 (23) at VAS 2 (infiltration of lidocaine in subcutaneous tissues at the initiation of the interventional pain procedure). As pain ratings were not dependent on the type of interventional pain procedure used (LESI, LFJI, or SIJI), type of procedure was not included as a cofactor. Age was not significantly correlated with lidocaine infiltration pain at any time point and was also not included.

Parameters from 3 classes (PSQ-E scores, clinical pain characteristics, and psychological cofactors of pain) were tested for prediction of lidocaine infiltration pain. In a first step, zeroorder correlations were calculated (Table 4). From each class, the variable with the highest (significant or nonsignificant) zeroorder correlation to lidocaine infiltration pain was selected for linear regression analysis. Only 1 variable from each class was selected because of significant collinearity of parameters within classes. For pain ratings at VAS 1, linear regression analysis with stepwise selection of PSQ-E-minor, Roland-Morris score, and PCS score as independent variables identified only PSQE-minor as predictor $(r=0.26, P<0.01$, Fig. 1A). For pain ratings at VAS 2 , linear regression analysis with stepwise selection of PSQ-E-minor, BPI interference score, and PCS score as independent variables identified PSQ-E-minor as the strongest

TABLE 3. Correlations Between PSQ-E Scores, Psychological Measures, and Clinical Pain Characteristics

\begin{tabular}{llcc}
\hline & PSQ-E-Total & PSQ-E-Minor* & PSQ-E-Moderate \\
\hline HADS-D & 0.05 & 0.05 & 0.03 \\
HADS-A & 0.14 & 0.13 & 0.12 \\
PCS & $0.32 \dagger$ & $0.33 \dagger$ & $0.28 \dagger$ \\
$\begin{array}{l}\text { Duration of } \\
\text { pain, mo }\end{array}$ & 0.15 & 0.17 & 0.10 \\
BPI pain score & $0.25 \S$ & 0.24 & 0.22 \\
$\begin{array}{l}\text { BPI interference } \\
\quad 0 \text { score }\end{array}$ & 0.15 & 0.16 & 0.11 \\
$\begin{array}{l}\text { Roland-Morris } \\
\text { score }\end{array}$ & 0.21 & 0.22 & 0.17 \\
\hline
\end{tabular}

$\mathrm{n}=136$. Pearson $r$ is given.

* Significance levels of correlations with PSQ-minor were confirmed using a nonparametric test (seen methods).

$\dagger P<0.001$.

$\ddagger P<0.01$.

$\S P<0.05$ after Bonferroni correction.
TABLE 4. Correlations Between Procedural Pain Ratings and Possible Predictors of Procedural Pain

\begin{tabular}{lccc}
\hline & & \multicolumn{2}{c}{ Pain Rating } \\
\cline { 3 - 4 } & & VAS 1* & VAS 2* \\
\hline \multirow{3}{*}{ PSQ } & $\mathrm{n}$ & 133 & 131 \\
& PSQ-E-total & $0.23 \dagger$ & $0.33 \dagger$ \\
& PSQ-E-minor c & $0.26 \dagger$ & $0.34 \dagger$ \\
Clinical pain & PSQ-E-moderate & $0.19 \dagger$ & $0.29 \dagger$ \\
characteristics & Duration of pain, mo & -0.03 & -0.01 \\
& BPI pain score & 0.16 & $0.25 \dagger$ \\
& BPI interference score & 0.14 & $0.27 \S$ \\
Psychological & Roland-Morris score & $0.18 \dagger$ & $0.25 \dagger$ \\
cofactors of pain & HADS-D & 0.15 & 0.11 \\
& HADS-A & 0.07 & 0.11 \\
& PCS & $0.21 \dagger$ & $0.24 \dagger$ \\
\hline
\end{tabular}

Total $\mathrm{n}=136$. Some patients did not give valid pain ratings at VAS 1 and VAS 2. Pain rating at VAS 3 was only introduced in the second half of the study. Pearson $r$ is given.

* Significance levels of correlations with PSQ-minor, VAS 1 and VAS 2 were confirmed using a nonparametric test (seen methods). Bonferroni adjustment was not used because the purpose of the zero-order correlations in the present list was only to select variables to enter linear regression analysis.

$\dagger P<0.05$.

$\ddagger P<0.001$.

$\S P<0.01$.

predictor (model 1: $r=0.34, P<0.001$, Fig. $1 \mathrm{~B} 1$ ), and the prediction was significantly enhanced by further inclusion of BPI interference score (model 2: $\beta_{\text {PSQ-E-minor }}=0.31, P<0.001$; $\beta_{\mathrm{BPI}}$ interference score $=0.22, P<0.01$; total $r=0.40, P<0.001$, Fig. 1B2).

We further tested if sex contributed to prediction of lidocaine infiltration pain. However, there were no significant sex differences in pain ratings at VAS 1 and VAS 2 .

\section{DISCUSSION}

The most important result of the present study was that it demonstrated a correlation between the PSQ-E and clinically relevant evoked pain ratings. Prediction of lidocaine infiltration pain was further improved when information from the BPI was added to the analysis. Hence, not only did this study validate the PSQ-E as a measure of pain sensitivity equivalent to the original German version (PSQ-G), but it also suggests that use of 2 brief questionnaires (PSQ and BPI) should have clinical utility in predicting those patients with high pain sensitivity. This predictive value is important because it makes possible the identification of high sensitivity before patients undergo a procedure, influencing clinical decisions.

Specifically, the properties of PSQ-E scores obtained from chronic back pain patients in the present study are similar to those previously obtained in a group of mixed chronic pain patients $(n=134)$ with the original German version. ${ }^{10}$ In the German study, PSQ-G scores were 4.0 (1.7) (PSQ-G-total), 2.9 (1.5) (PSQ-G-minor), and 5.1 (2.0) (PSQ-G-moderate). ${ }^{10}$ These values are similar to those found with the PSQ-E in the present study. In terms of psychological cofactors of pain perception, the PSQ-E exhibits highest correlations with the 

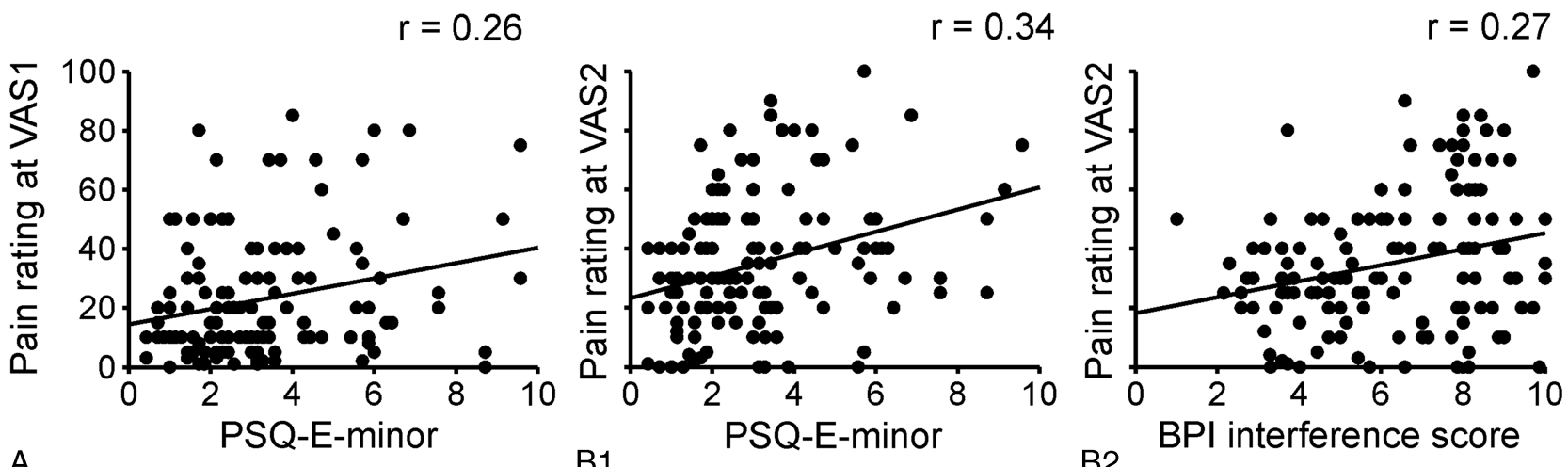

FIGURE 1. Correlations between pain ratings at VAS 1 and VAS 2 and predictors identified by linear regression. A, Pain rating at VAS 1. B, Pain rating at VAS 2. Pearson $r$ is given.

pain-specific measure (PCS) and nonsignificant correlations with measures of anxiety and depression (Table 3 ). These results are comparable to those obtained with the PSQ-G, both in healthy subjects ${ }^{9}$ and in chronic pain patients. ${ }^{10}$ These results also support convergent validity (correlation with pain-specific measure) and divergent validity (no correlation with nonpainspecific measure) of the PSQ-E.

Regarding the relation with clinical pain characteristics, similar to previous results with the PSQ-G in chronic pain patients, ${ }^{10}$ there was no correlation between PSQ-E and chronic pain duration (Table 3). There was a significant correlation of PSQ-E scores with BPI pain scores, ${ }^{10}$ similar to the correlation between clinical pain intensity ratings and PSQ-G scores in the German study $(r=0.18)$. Similar to previous studies using the PSQ-G in healthy subjects ${ }^{9}$ and chronic pain patients, ${ }^{10}$ there were no significant differences in PSQ-E scores between males and females in the current study. Similar results have been found for the PSQ-G, regarding correlations with results of experimental pain testing ${ }^{9}$ and for PSQ-G differences between healthy subjects and chronic pain patients. ${ }^{10}$

In summary, both mean values and correlations with different pain-related variables are very similar between PSQ-E and PSQ-G in chronic pain patients, and PSQ-minor subscores seem to be slightly superior to PSQ-total scores in both versions. These results suggest that the English translation shares reliability and validity with the original German version and that results obtained with the PSQ-E are directly comparable to those obtained with the PSQ-G.

\section{Prediction of Lidocaine Infiltration \\ Pain by the PSQ-E}

Pain during infiltration of local anesthetic in the hand or back (VAS 1 and VAS 2, respectively) was predicted by PSQE-minor scores with an $r$ around 0.3. As the PSQ is a measure of general pain perception, or "pain sensitivity," results would be expected to be similar to the prediction of procedural pain by experimental pain testing. Although there are to the best of our knowledge no reports attempting to predict pain during an interventional procedure by experimental pain testing, greater pain perception-when assessed preoperatively by advanced experimental pain testing - has been associated with greater persistent postoperative pain scores. ${ }^{6,34}$ Reported correlation coefficients for the prediction of postoperative pain by experimental pain testing have ranged between 0.1 and $0.5 . .^{35-39}$ Therefore, the prediction of lidocaine infiltration pain by the PSQ in the present study is within the expected range.
Pain during lidocaine infiltration in the back (VAS 2) marked the greatest level of lidocaine infiltration pain collected within the present study, and prediction of this parameter may, therefore, be most relevant from a clinical perspective.

\section{Application of the PSQ for the Pain Clinic Patient}

Interindividual variability in pain perception is large, both in healthy subjects and in chronic pain patients. ${ }^{10-12}$ Such differences in pain sensitivity seem to influence pain experiences, the development of chronic pain syndromes, and responses to analgesic treatment. ${ }^{4,40}$ Unfortunately, chronic pain patients exhibit a generalized enhancement of pain perception. ${ }^{7,41,42}$ The overlap in pain perception between healthy subjects and chronic pain patients is large so that strategies for identifying individuals with increased risk of sensitivity are needed. The present results show that the PSQ-E may be used to identify those patients.

\section{Limitations}

The study has several limitations that may have affected the correlation of the PSQ scores and procedural pain. Two clinicians performed the standardized lidocaine injection. This may have resulted in variability of this pain stimulus.

Another limitation relates to the low VAS 1 scores noted. Some patients may have experienced little or no pain during the pain stimulus of lidocaine infiltration if the means and standard deviations are considered; $47 \%$ of the patients had VAS 1 scores below or equal to 10 based on the results. This may have helped to reveal low and high pain sensitivities. It was felt to be meaningful to have the entire spectrum of responses because it was reflective of patients' responsiveness.

Combination of the PSQ with the BPI enhanced predictive power of a patient's lidocaine infiltration pain and sensitivity. Although clinical lore would suggest a correlation between sensitivity and a need for sedation, it will be up to the individual clinician to determine value of this measure of sensitivity for their practice. The typical pain clinician may prefer to only use 1 measure for clinical utility as opposed to 2 , which were needed from the results of the study. This was done by Cohen et $\mathrm{al}^{32}$ who used the standardized subcutaneous local anesthetic injection to predict response to epidural steroid injections. Use of this measure with PSQ and BPI could potentially allow for even greater predictive value in future studies. 


\section{CONCLUSIONS}

This study validated the English version of the PSQ, and, if coupled with the BPI, it demonstrated utility in predicting a patient's pain with lidocaine infiltration. The PSQ offers advantages in a high-volume clinic setting because it can be quickly administered and is noninvasive. Future studies may assess test-retest reliability. In the present study, we only included white patients for better comparability with the existing German PSQ data, but ongoing studies are currently seeking to determine the contributions that ethnicity may contribute to specific measures. Future research may also explore for correlations in PSQ scores and other factors known to alter pain sensitivity such as medication use.

\section{ACKNOWLEDGMENTS}

The authors thank Theresa Hensley and Terry Byars for the assistance with data entry.

\section{REFERENCES}

1. Hirsh AT, George SZ, Bialosky JE, Robinson ME. Fear of pain, pain catastrophizing, and acute pain perception: relative prediction and timing of assessment. J Pain. 2008;9:806-812.

2. Ip HY, Abrishami A, Peng PW, Wong J, Chung F. Predictors of postoperative pain and analgesic consumption: a qualitative systematic review. Anesthesiology. 2009;111:657-677.

3. Thibault P, Loisel P, Durand MJ, Catchlove R, Sullivan MJ. Psychological predictors of pain expression and activity intolerance in chronic pain patients. Pain. 2008;139:47-54

4. Edwards RR. Individual differences in endogenous pain modulation as a risk factor for chronic pain. Neurology. 2005;65:437-443.

5. Edwards RR, Sarlani E, Wesselmann U, Fillingim RB. Quantitative assessment of experimental pain perception: multiple domains of clinical relevance. Pain. 2005;114:315-319.

6. Granot M. Can we predict persistent postoperative pain by testing preoperative experimental pain? Curr Opin Anaesthesiol. 2009;22: $425-430$.

7. Giesecke T, Gracely RH, Grant MA, et al. Evidence of augmented central pain processing in idiopathic chronic low back pain. Arthritis Rheum. 2004;50:613-623.

8. Hastie BA, Riley JL 3rd, Robinson ME, et al. Cluster analysis of multiple experimental pain modalities. Pain. 2005;116:227-237.

9. Ruscheweyh R, Marziniak M, Stumpenhorst F, Reinholz J, Knecht S. Pain sensitivity can be assessed by self-rating: development and validation of the Pain Sensitivity Questionnaire. Pain. 2009;146:65-74.

10. Ruscheweyh R, Verneuer B, Dany K, et al. Validation of the pain sensitivity questionnaire in chronic pain patients. Pain. 2012;153:1210-1218

11. Edwards RR, Doleys DM, Lowery D, Fillingim RB. Pain tolerance as a predictor of outcome following multidisciplinary treatment for chronic pain: differential effects as a function of sex. Pain. 2003;106:419-426.

12. Edwards RR, Haythornthwaite JA, Tella P, Max MB, Raja S. Basal heat pain thresholds predict opioid analgesia in patients with postherpetic neuralgia. Anesthesiology. 2006;104:1243-1248.

13. Williamson A, Hoggart B. Pain: a review of three commonly used pain rating scales. J Clin Nurs. 2005;14:798-804.

14. Hjermstad MJ, Fayers PM, Haugen DF, et al. Studies comparing Numerical Rating Scales, Verbal Rating Scales, and Visual Analogue Scales for assessment of pain intensity in adults: a systematic literature review. J Pain Symptom Manage. 2011;41:1073-1093.
15. Cleeland C. Measurement of pain by subjective report. In: Chapman $\mathrm{CR}$, Loeser JD, eds. Issues in Pain Measurement. New York, NY: Raven Press; 1989:391-403.

16. Riddle DL, Stratford PW, Binkley JM. Sensitivity to change of the Roland-Morris Back Pain Questionnaire: part 2. Phys Ther. 1998:78:1197-1207.

17. Roland M, Morris RA. Study of the natural history of back pain. Part I: development of a reliable and sensitive measure of disability in low-back pain. Spine (Phila Pa 1976). 1983;8:141-144.

18. Roland M, Morris RA. Study of the natural history of low-back pain. Part II: development of guidelines for trials of treatment in primary care. Spine (Phila Pa 1976). 1983;8:145-150.

19. Bjelland I, Dahl AA, Haug TT, Neckelmann D. The validity of the Hospital Anxiety and Depression Scale. An updated literature review. J Psychosom Res. 2002;52:69-77.

20. Sullivan MJL, Bishop SR. The pain catastrophizing scale: development and validation. Psychol Assess. 1995;7:524-532.

21. Beaton DE, Bombardier C, Guillemin F, Ferraz MB. Guidelines for the process of cross-cultural adaptation of self-report measures. Spine (Phila Pa 1976). 2000;25:3186-3191.

22. Nakayama M, Munemura Y, Kanaya N, Tsuchida H, Namiki A. Efficacy of alkalinized lidocaine for reducing pain on intravenous and epidural catheterization. J Anesth. 2001;15:201-203.

23. Burgher SW, McGuirk TD. Subcutaneous buffered lidocaine for intravenous cannulation: is there a role in emergency medicine? Acad Emerg Med. 1998;5:1057-1063.

24. Burns CA, Ferris G, Feng C, Cooper JZ, Brown MD. Decreasing the pain of local anesthesia: a prospective, double-blind comparison of buffered, premixed $1 \%$ lidocaine with epinephrine versus $1 \%$ lidocaine freshly mixed with epinephrine. $J$ Am Acad Dermatol. 2006;54:128-131.

25. Xia Y, Chen E, Tibbits DL, Reilley TE, McSweeney TD. Comparison of effects of lidocaine hydrochloride, buffered lidocaine, diphenhydramine, and normal saline after intradermal injection. J Clin Anesth. 2002;14:339-343.

26. Younis I, Bhutiani RP. Taking the 'ouch' out - effect of buffering commercial xylocaine on infiltration and procedure pain - a prospective, randomised, double-blind, controlled trial. Ann R Coll Surg Engl. 2004;86:213-217.

27. Steinbrook RA, Hughes N, Fanciullo G, Manzi D, Ferrante FM Effects of alkalinization of lidocaine on the pain of skin infiltration and intravenous catheterization. J Clin Anesth. 1993;5:456-458

28. George RB, Habib AS, Allen TK, Muir HA. Brief report: a randomized controlled trial of Synera versus lidocaine for epidural needle insertion in labouring parturients. Can J Anaesth. 2008;55:168-171.

29. Zsigmond EK, Darby P, Koenig HM, Goll EF. Painless intravenous catheterization by intradermal jet injection of lidocaine: a randomized trial. J Clin Anesth. 1999;11:87-94.

30. Cohen SP, Christo PJ, Wang S, et al. The effect of opioid dose and treatment duration on the perception of a painful standardized clinical stimulus. Reg Anesth Pain Med. 2008;33:199-206.

31. Manabat ER, Pujol LA, Hunt P, Wang D. Judging pain sensitivity with subcutaneous lidocaine injections. Pain Med. 2011;12:668-672.

32. Cohen SP, Mao J, Vu T, et al. Does pain score in response to a standardized subcutaneous local anesthetic injection predict epidural steroid injection outcomes in patients with lumbosacral radiculopathy? A prospective correlational study. Pain Med. 2013;14:327-335.

33. Harris RJ. A Primer of Multivariate Statistics. Mahwah, NJ: Lawrence Erlbaum Associates; 2001.

34. Werner MU, Mjobo HN, Nielsen PR, Rudin A. Prediction of postoperative pain: a systematic review of predictive experimental pain studies. Anesthesiology. 2010;112:1494-1502. 
35. Bisgaard T, Klarskov B, Rosenberg J, Kehlet H. Characteristics and prediction of early pain after laparoscopic cholecystectomy. Pain. 2001;90:261-269

36. Bisgaard T, Rosenberg J, Kehlet H. From acute to chronic pain after laparoscopic cholecystectomy: a prospective follow-up analysis. Scand J Gastroenterol. 2005;40:1358-1364.

37. Granot M, Lowenstein L, Yarnitsky D, Tamir A, Zimmer EZ. Postcesarean section pain prediction by preoperative experimental pain assessment. Anesthesiology. 2003;98:1422-1426.

38. Hsu YW, Somma J, Hung YC, Tsai PS, Yang CH, Chen CC. Predicting postoperative pain by preoperative pressure pain assessment. Anesthesiology. 2005;103:613-618.
39. Pan PH, Coghill R, Houle TT, et al. Multifactorial preoperative predictors for postcesarean section pain and analgesic requirement. Anesthesiology. 2006;104:417-425.

40. Nielsen CS, Staud R, Price DD. Individual differences in pain sensitivity: measurement, causation, and consequences. J Pain. 2009;10:231-237.

41. Maixner W, Fillingim R, Booker D, Sigurdsson A. Sensitivity of patients with painful temporomandibular disorders to experimentally evoked pain. Pain. 1995;63:341-351.

42. Schoenen J, Bottin D, Hardy F, Gerard P. Cephalic and extracephalic pressure pain thresholds in chronic tension-type headache. Pain. 1991;47:145-149. 